\title{
Gubernamentalidad liberal, evaluación y verdad: Sobre el uso politico del conocimiento técnico
}

\author{
Liberal governmentality, evaluation and truth: On the political \\ use of technical knowledge
}

\author{
Ángel Rivero Recuenco \\ Universidad de Alcalá (Madrid) \\ arrecuen@hotmail.com
}

Recibido: 05.052019

Aceptado: 11.05.2020

\section{RESUMEN}

La evaluación de intervenciones públicas constituye una disciplina técnica cuya finalidad es informar la toma de decisiones gubernamentales. Por ello, resulta especialmente relevante para analizar el problema del uso político del conocimiento. En este sentido, la historia de esta disciplina pone de manifiesto el enfrentamiento entre paradigmas metodológicos en cuya pugna están en juego distintos modos de aplicar los resultados de las evaluaciones. Asimismo, se evidencian las dificultades y distorsiones de esta puesta en práctica, el incumplimiento persistente de los fines asignados a la evaluación. Ello plantea una paradoja: el sentido último de la evaluación es incrementar la eficacia y la utilidad de la acción gubernamental; sin embargo, comparece como una práctica inútil, sin uso efectivo. El presente artículo indaga en las razones de esta paradoja desde una doble perspectiva, constituida por la concepción foucaultiana del acoplamiento entre saberes y dispositivos de gobierno, y las reflexiones de Hannah Arendt sobre la relación entre verdad y política en las sociedades contemporáneas.

\section{PALABRAS CLAVE}

Gubernamentalidad Liberalismo Gerencialismo Evaluación de políticas públicas. 


\begin{abstract}
The evaluation of public interventions is a technical discipline whose purpose is to inform government decision-making. Therefore, it is especially relevant in order to analyze the problem of the political use of knowledge. In this sense, the history of this discipline shows the confrontation between methodological paradigms in whose struggle different ways of implementing the results of evaluations are at stake. Likewise, the difficulties and distortions of this implementation are evidente: the persistent non-compliance of the purposes assigned to the evaluation. This raises a paradox: the ultimate meaning of evaluation is to increase the effectiveness and utility of government action; however, it appears as a useless practice, a practice without effective use. The present article explores the reasons for this paradox from a double perspective, constituted by the Foucaultian conception of the coupling between knowledge and government devices, and the reflections of Hannah Arendt on the relationship between truth and politics in contemporary societies.
\end{abstract}

\title{
KEY WORDS
}

Governmentality Liberalism Technocracy Public policy evaluation.

\section{INTRODUCCIÓN}

La evaluación es un saber instrumental llamado a proyectarse sobre diversos tipos de intervención planificada, con el fin de producir juicios de valor sobre la utilidad y conveniencia de estas. En su aplicación al ámbito de las políticas públicas, tales intervenciones adoptan la forma de programas y servicios, principalmente de carácter "social": programas educativos, servicios sociosanitarios, planes de empleo o de lucha contra la pobreza..., que conforman los puntos posibles de aplicación del saber evaluativo. Los efectos esperados de este saber están bien definidos en el plano teórico: racionalización presupuestaria, reordenación de políticas, justificación de decisiones en términos de eficacia y eficiencia.

La historia de la evaluación muestra, sin embargo, una persistente contradicción: el cumplimiento de tales fines teóricos es, en la práctica, irrelevante. La evaluación, cuyo sentido último es la utilidad, comparece, de este modo, como una actividad ineficaz: un discurso y una práctica sin uso efectivo. El hecho de que esta suerte de paradoja se dé en el ámbito de la evaluación, una disciplina expresamente orientada a proporcionar al gobierno un saber técnico que lo oriente, focaliza el problema del uso del conocimiento en su mismo núcleo, allí donde, por definición, esa contradicción no debería darse. Por ello, lo que muestra la historia de la evaluación no es un problema específico del campo evaluativo, sino un síntoma especialmente revelador de la problemática de fondo que afecta 
a nuestros supuestos sobre el lugar del conocimiento técnico en la toma de decisiones políticas.

En este artículo tratamos de esclarecer los motivos de esta contradicción. En primer lugar, describimos las condiciones históricas de formación del discurso evaluativo. En segundo lugar, asumiendo como perspectiva la genealogía del liberalismo de Michel Foucault (2009), mostramos la relación entre dicho discurso y las tecnologías liberales de gobierno. A continuación, constatamos la persistente ineficacia de las evaluaciones respecto a sus fines teóricos, y respecto a los propios principios de la gubernamentalidad liberal. Finalmente, tratamos de dar razón de esta aparente anomalía, a partir de dos marcos teóricos diferenciados: la concepción foucaultiana de la relación entre saberes y dispositivos de gobierno proporciona una primera aproximación explicativa a la paradoja de la evaluación -y, más allá de esta, a la cuestión general del uso político del conocimiento técnico; las reflexiones de Hannah Arendt $(2015,2016)$ acerca de la relación entre verdad y política desarrollan y completan, de forma radical, la hipótesis foucaultiana ${ }^{1}$.

\section{MARCO HISTÓRICO: LA FORMACIÓN DEL DISCURSO EVALUATIVO}

\subsection{La génesis norteamericana}

Es en los Estados Unidos, a principios del siglo pasado, donde el término evaluación aparece, constituyéndose de forma positiva en objeto de discurso. Un objeto cuyo campo de emergencia es la práctica educativa y su punto de aplicación, la medición del aprendizaje. Diferentes líneas coinciden para dar lugar a la formación de un discurso sobre la evaluación del aprendizaje: el desarrollo de las ciencias sociales, el movimiento del gerencialismo científico, la expansión de las políticas educativas, el auge de la psicometría. A partir de estas convergencias y sobre ese campo de emergencia inicial, el discurso evaluativo se proyectará, muchos años después, en la década de 1960, sobre el ámbito de la política social².

En la primera etapa de formación del discurso evaluativo, desde principios del siglo XX hasta la década de 1930, el problema a resolver es la medición del aprendizaje por medio de test psicométricos. En su segunda etapa, denominada descriptiva o de evaluación de objetivos, a partir de los trabajos de Ralph Tyler

${ }^{1}$ El artículo tiene como punto de partida nuestra investigación sobre la evaluación de intervenciones públicas en España, desarrollada como tesis doctoral y defendida en junio de 2015 en la Facultad de Ciencias Políticas y Sociología de la UNED. En esta investigación se apuntan ya los problemas del uso político del conocimiento y de las relaciones entre verdad y política. En el presente artículo profundizamos en estas cuestiones, incorporando nuevas perspectivas de análisis.

2 Sobre las distintas fases del desarrollo de la evaluación en los Estados Unidos, Cfr. Cronbach (1963), Stake (1967), McLaughlin (1975), House (1979, 2000) y Guba y Lincoln (1986, 1989). Análisis histórico-comparativos acerca de la situación de la evaluación en distintos países pueden consultarse en Furubo et al. (2002) y Urteaga (2010). 
(Smith y Tyler 1942; Tyler 1950), se produce una ampliación de su objeto: se pasa de la medición de variables de aprendizaje en sujetos individuales a la valoración de los programas educativos en sí mismos. La redefinición del punto de aplicación del saber evaluativo hacia una entidad de carácter más general, el programa, permite su "emancipación" del campo educativo, su constitución como saber autónomo. Esto es lo que va a permitir a la evaluación proyectarse sobre cualquier ámbito de intervención planificada, expandir sus métodos más allá del entorno educativo. La consideración de la forma-programa como objeto es, así, condición de posibilidad del desarrollo del saber evaluativo, de su expansión discursiva e institucional.

En este sentido, la institucionalización político-administrativa de la evaluación va a acontecer en la década de 1960, en el marco de los programas federales de guerra contra la pobreza desarrollados por la Administración Johnson; programas cuyo impacto había que determinar científicamente. Este es el contexto en el que tiene lugar la expansión administrativa de la evaluación. Un contexto caracterizado por una suerte de optimismo tecnocrático, de confianza en la capacidad de las tecnologías de planificación y evaluación para resolver las desigualdades de la sociedad norteamericana. El modelo hegemónico en esta fase de expansión e institucionalización de la evaluación (y, en realidad, a lo largo de toda su historia) es el modelo de análisis de sistemas, cuya concreción más conocida será el llamado enfoque sistemático (Rivlin 1971, Rossi et al. 1979). Este enfoque se va a erigir en paradigma de aplicación del gerencialismo en el campo evaluativo ${ }^{3}$.

El enfoque sistemático constituye un modelo orientado a la medición, aunque más sofisticado que el enfoque tyleriano de evaluación de objetivos, y con implicaciones políticas de mayor alcance. Alice Rivlin (1971), en el texto fundacional del enfoque sistemático, Systematic Thinking for Social Action, parte de una constatación relativa al funcionamiento de los servicios sociales federales: la dispersión de sus modalidades organizativas dificultaba conocer adecuadamente sus resultados, impedía un conocimiento sistemático de lo que producían dichos servicios. Para ello, habría que establecer una función de producción que especificase relaciones estables entre las variables de entrada en el sistema (inputs) y sus resultados (outputs). El medio para obtener ese conocimiento lo proporcionarían la estadística y la aplicación de modelos cuasi-experimentales de investigación. Solo de este modo, y solo mediante una gerencia muy centralizada, se obtendría la información necesaria para mejorar la eficacia y la eficiencia de los servicios sociales. En su aplicación a los programas sociales federales de guerra contra la pobreza, constituidos, a su vez, por miles de proyectos locales, el enfoque sistemático se concretó, a partir de 1966, en el diseño de modelos que

${ }^{3}$ Un enfoque de evaluación es considerado gerencial cuando sus objetivos y criterios de valoración son determinados por la tecnoestructura que administra la intervención evaluada. Los resultados de la evaluación revierten únicamente a dicha tecnoestructura (no hay participación de otros actores, ni difusión de resultados, más allá de los límites de la gerencia). Autores como Scriven (1973), Guba y Lincoln (1989) y House y Howe (2001) plantearon alternativas a este tipo de enfoques, como veremos enseguida. 
permitieran medir y comparar series limitadas de indicadores de resultado según diferentes tipos de proyecto. Cada modelo incluía test pre y postratamiento, un método de estimación de incrementos en la línea base (situación en ausencia de proyecto) y procedimientos de conversión de los resultados locales en equivalentes de curva normal, con el fin de permitir la integración de datos y las comparaciones entre proyectos.

\subsection{Rupturas: Epistemología, metodología y política}

A pesar de la sofisticación estadística y los recursos invertidos en las evaluaciones de los programas federales de guerra contra la pobreza, sus resultados fueron insatisfactorios, ya que no permitieron inferir patrones de relación claros entre tipos de proyecto y efectos producidos. Las evaluaciones fueron muy cuestionadas por su reduccionismo estadístico, por la inadecuación de sus instrumentos de medición y por la falta de fundamento empírico de los tipos de proyecto objeto de comparación ${ }^{4}$. Ante esta decepción, y en el contexto de las discusiones en torno al uso y la validez de las perspectivas cuantitativa y cualitativa en investigación social, los presupuestos epistemológicos y los criterios metodológicos del enfoque sistemático serán objeto de múltiples cuestionamientos en el campo evaluativo. Así, en la década de 1970, este enfoque se constituye en el referente contra el que se van a forjar diferentes modelos de evaluación alternativos. Destacamos tres:

La evaluación libre de objetivos (Goal Free Evaluation) de Michael Scriven (1973), que rechaza la centralidad de la perspectiva gerencial para determinar los valores-criterio en la evaluación de una intervención: el evaluador debe ignorar los propósitos de gerentes, promotores o financiadores respecto a las intervenciones sometidas a evaluación. La perspectiva relevante para formular juicios sobre el valor de las intervenciones evaluadas debe formarse a partir de las necesidades expresadas por los destinatarios de dichas intervenciones. De este modo, el evaluador se libera del sesgo constituido por los intereses específicos de las gerencias. En esta línea, Scriven $(1972,1976)$ propone una noción cualitativa de objetividad basada en la calidad de las observaciones y la ausencia de sesgos, opuesta a la idea cuantitativa de objetividad propia del enfoque sistemático, fundamentada esta en el número de observaciones replicables y el acuerdo intersubjetivo entre observadores. Para Scriven, la objetividad cuantitativa encierra, en la práctica, una consecuencia perversa: la preocupación por la fiabilidad de los instrumentos de medición deja en segundo plano la cuestión, más fundamental, de su validez.

El enfoque de estudio de casos de Robert Stake (1978), quien reivindica un mayor descenso del evaluador al campo, la recuperación de las experiencias de

${ }^{4}$ El caso paradigmático fue el de las sucesivas evaluaciones del programa Follow Thruogh, que agrupaba treinta mil proyectos locales. MacLaughlin (1975) y House $(1979,2000)$ proporcionan una detallada descripción de estas evaluaciones. 
los diferentes sujetos afectados por las evaluaciones, una relación más sensible hacia los llamados stakeholders (beneficiarios y damnificados por las intervenciones evaluadas, así como sus técnicos y gestores). Ello va a desembocar en la puesta en práctica de evaluaciones naturalistas de orientación eminentemente cualitativa y, en definitiva, en la constitución de un enfoque de evaluación basado en estudios de caso de inspiración etnográfica, articulados mediante la participación y la negociación de perspectivas. En este enfoque, el evaluador se constituye en mediador: coordina y canaliza los flujos de información, modera las interacciones y negociaciones de sentido inherentes al proceso evaluativo, se abstiene de emitir interpretaciones valorativas y recomendaciones. Los grupos participantes en este proceso harán el uso que entiendan más apropiado de los resultados de la evaluación.

El Connoisseurship Model de Elliot Eisner (1979), basado en el conocimiento tácito del experto. Para Eisner, el punto de vista legitimado para valorar es el del experto en el ámbito temático (educación, empleo, sanidad...) en el que se inscribe la intervención evaluada. El evaluador, en tanto que experto sectorial, evalúa dicha intervención de modo similar a como un crítico valoraría una obra de arte. La epistemología subjetivista basada en el conocimiento tácito experto se contrapone, así, a la epistemología objetivista y al conocimiento explícito, altamente formalizado, de los enfoques basados en el modelo del análisis de sistemas.

Las rupturas epistemológicas y metodológicas de la década de 1970, en especial el enfoque de estudio de casos, van a abrir la puerta al desarrollo de una tendencia interpretativa y dialógica en el campo de la evaluación. Una tendencia que, como veremos enseguida, será especialmente productiva en las décadas siguientes, alcanzando su forma teóricamente más elaborada en el enfoque constructivista-respondiente de Guba y Lincoln (1986, 1989).

Pero las rupturas de los años '70 no se dieron solo en los planos epistemológico y metodológico. También tuvieron implicaciones políticas. A la confianza tecnocrática de los años '60 le sigue, en la década de 1970, la decepción: se constata que los programas de intervención social no logran modificar la realidad tan mecánicamente como se esperaba; además, el uso de las evaluaciones se revela, en la práctica, muy limitado. Estas decepciones desembocaron en una crítica política de los supuestos y el sentido general de la evaluación. Los trabajos de Carol Weiss resultan especialmente indicativos al respecto: sobre la base de un extenso estudio acerca del uso de la evaluación por los gestores federales, estatales y locales en el campo de la salud mental, Weiss $(1980,1987)$ cuestionó el supuesto racional convencional de que la evaluación proporcionaba una base de carácter instrumental para la acción política. Las conclusiones de este estudio señalaron que, aunque las evaluaciones daban lugar a cierta gama de usos, se utilizaban más bien como base para legitimar posturas. Las evaluaciones (y en general las investigaciones sociales) no constituían una fuente sólida para dirigir los procesos decisionales, sino que tendían a aceptar las premisas políticas de los programas evaluados, otorgando a estos "un aura de legitimidad" (Weiss 1987: 49). 
En este contexto de decepción y crítica política, desde finales de la década de 1980 se van a intensificar los debates y las rupturas teórico-metodológicas en el campo evaluativo. La más notable, y también la más radical, es la representada por el enfoque constructivista-respondiente de Guba y Lincoln, que constituye una radicalización del enfoque de estudio de casos de Robert Stake. Partiendo de premisas epistemológicas declaradamente relativistas, Guba y Lincoln conciben el objeto de una evaluación (el evaluando) como algo que se ha de construir discursivamente a lo largo del proceso evaluativo. El medio para tal construcción son los llamados círculos hermenéuticos-dialécticos, donde los diferentes grupos afectados por la evaluación (los stakeholders) van a revisar, en un proceso continuo, sus propias percepciones e interpretaciones del evaluando, llegando progresivamente a nuevas y más elaboradas apreciaciones de sus debilidades y fortalezas. La función principal del evaluador es, simplemente, coordinar y estimular este proceso (Guba y Lincoln 1989: 71-74).

En perspectiva política, el enfoque constructivista-respondiente inscribe la evaluación en un campo de relaciones de poder, cuyo juego de fuerzas determina sus usos. Guba y Lincoln constatan críticamente la asimetría que caracteriza el desarrollo las evaluaciones: el gerencialismo dominante excluye de la participación tanto a los beneficiarios como a los posibles perjudicados por las intervenciones evaluadas, privándoles de la posibilidad de defenderse de las consecuencias de la evaluación. Todas las decisiones evaluativas se adoptan en el círculo exclusivo de la administración del programa evaluado. La evaluación constructivista se propone como respuesta a esta situación excluyente e injusta: una forma de superar, por medio de la participación, las distorsiones y limitaciones en los usos de la evaluación. Para Guba y Lincoln, a mediados de la década de 1980, la acción estaba "singularmente ausente en la mayor parte de las evaluaciones". Su enfoque, "con su acento en la implicación de stakeholders", se comprometía a "estimular la acción de un modo y en un grado sin precedentes" (Ibid.: 249).

Una década después, House y Howe (2001) incidirán en el mismo problema, proponiendo un modelo democrático-deliberativo como forma de superar el déficit de usos de la evaluación o su uso perverso con fines preconcebidos de legitimación. Sin embargo, para estos autores, el problema no radicaba solo en los enfoques gerencialistas de evaluación. El estudio de casos y la evaluación constructivista-respondiente tampoco aseguraban una adecuada representación de los intereses y necesidades de los grupos sociales más vulnerables. En estos enfoques participativos, la atribución al evaluador de un rol de mero facilitador de los intercambios comunicativos entre stakeholders favorecía que fueran los intereses de los grupos más poderosos los que, finalmente, se reprodujeran. Para superar esta parálisis política, House y Howe establecieron un marco de referencia "para juzgar las evaluaciones basándonos en su potencial de deliberación democrática" (House y Howe 2001: 140). Dicho marco de referencia estaría constituido por tres criterios: inclusión, diálogo y deliberación, concebida esta como un proceso racional y empíricamente fundamentado de argumentación e interacción comunicativa: "un proceso cognitivo, fundado en la razón, las pruebas y los principios del razonamiento válido" (Ibid.: 145). El evaluador ya no es 
un mero animador de este proceso, sino su garante: encauza el diálogo, asegura una distribución equitativa de la participación y distingue "las afirmaciones autorizadas de las autoritarias" (Ibid.: 131). De este modo, la acción política se vería promovida sobre la base de un conocimiento adecuadamente construido y fundamentado. Un conocimiento, proporcionado por la evaluación y controlado por el evaluador, que contrarrestaría las dinámicas de desequilibrio de poder, manipulación y confusión generadas por efecto de la publicidad y la comunicación de masas, permitiendo a los ciudadanos hacerse "juicios informados de los problemas públicos, sin sentirse defraudados, engañados ni confundidos" (Ibid.).

Con el desarrollo de los enfoques dialógicos, participativos y democráticos de los años '80 y '90, el campo de la evaluación alcanza lo que podríamos considerar su "nivel de saturación" teórico-metodológico. Los debates del siglo XXI son debates heredados, y ya muy atenuados, de las controversias y rupturas sucedidas entre los años ' 70 y ' 90 del siglo pasado en el ámbito norteamericano. Con todo, más allá de estas rupturas y de la emergencia de modelos de evaluación alternativos, es importante dejar claro un hecho: la persistencia en el campo evaluativo de una marcada hegemonía gerencial. A pesar de las críticas de que fue objeto, lo cierto es que el paradigma sistemático ha constituido una referencia metodológica fundamental para el desarrollo de enfoques evaluativos de gestión por objetivos y análisis coste-resultado; enfoques de inequívoca filiación empresarial, dominantes en los dispositivos gubernamentales de evaluación, no sólo en Norteamérica, sino también, a partir de la década de 1990, en Europa ${ }^{5}$. Volveremos sobre este punto en el siguiente apartado.

\section{MARCO POLÍTICO: GUBERNAMENTALIDAD LIBERAL $Y$ EVALUACIÓN}

Es obvio que la evaluación, a la vez que instrumento de saber, constituye un medio para ejercer el poder. Depende, de forma más pregnante si cabe que en otros saberes, de una determinada tecnología de gobierno; esto es, dicho en términos foucaultianos, depende de una gubernamentalidad específica. Gubernamentalidad como soporte y condición política de posibilidad de un saber, la evaluación, que, a su vez, se configura como instrumento de esa práctica de gobierno. Nos preguntamos, entonces, qué forma de gubernamentalidad se hace visible en el discurso evaluativo.

La gubernamentalidad liberal, el régimen de veridicción introducido por la economía política a partir del siglo XVIII, y la evaluación, como saber instrumental y tecnología de gobierno, están estrechamente ligados. Esta apreciación, inspirada en la genealogía del liberalismo de Michel Foucault (2009), constituye el eje de nuestra argumentación. Enunciada brevemente, reza como sigue:

${ }^{5}$ Sobre la recepción europea del discurso evaluativo y, en general, sobre la situación de la evaluación a principios de este siglo, Cfr. Merino (2010) y Urteaga (2010). 
En el desarrollo del saber evaluativo encontramos en obra dos rasgos básicos atribuidos por Foucault a la gubernamentalidad liberal:

1. La utilidad y el interés como principios de racionalidad de la acción de gobierno.

2. La distinción éxito/fracaso y el examen de los efectos como formas de establecer la verdad de tal acción gubernamental.

Estos dos rasgos están estrechamente vinculados. La limitación del poder gubernamental es el lazo que las une. Son condiciones básicas de las tecnologías liberales de gobierno y, también, mucho tiempo después de su aparición, harán posible la formación del discurso y la práctica evaluativas.

\subsection{Utilidad e interés}

En sus aplicaciones concretas, las evaluaciones producen juicios relativos a las consecuencias de la acción de gobierno sobre la base de una observación técnica y empírica, de una razón científica. Básicamente, en esa aplicación del saber evaluativo, se trata de ajustar dos conjuntos de elementos: el de la acción de gobierno, con sus objetivos, sus procesos y sus costes, por un lado, y el de los efectos derivados de dicha acción, por otro. Los procesos deben ser funcionales, coadyuvar al logro de los objetivos de la intervención gubernamental, contribuir eficazmente a la producción de efectos. Estos, por su parte, deben justificar los costes de esa intervención. La evaluación verifica el ajuste entre estos elementos, objetivando técnicamente la acción de gobierno.

De lo anterior se deriva que la utilidad, la funcionalidad, la eficacia, en definitiva, constituyen las claves de inteligibilidad del discurso y la práctica de la evaluación. El discurso evaluativo eleva el grado de formalización del valor clásico de la utilidad. Lo operacionaliza por medio del criterio de eficacia y su derivada económica, la eficiencia. Encontramos asentada la centralidad de estos criterios en el modelo de evaluación de objetivos de Tyler y en su derivación más acabada, el enfoque sistemático, que, más allá de las rupturas que acontecen en el campo de la evaluación, permanecerá como modelo de referencia de la práctica evaluativa tanto en Estados Unidos como en Europa.

Pero, mucho antes de que el discurso evaluativo se constituya, la utilidad aparece como el problema fundamental del radicalismo político inglés. En esta concepción del arte de gobernar, el término radical designa una posición frente al poder estatal: "la posición consistente en plantear de continuo al gobierno (...) la cuestión de su utilidad o su falta de utilidad" (Foucault 2009: 54). El utilitarismo aparece, entonces, desde el siglo XVIII, como una forma de delimitar la esfera de competencia gubernamental en términos de utilidad. Una razón de gobierno que se encuentra ligada a otro principio: el interés, que se constituye, en los comienzos del liberalismo, desde sus primeros fundamentos en la filosofía empírica inglesa, en el criterio de la utilidad. Pero tal interés ya no es el de la razón de Estado, "el del Estado íntegramente referido a sí mismo" (Ibid.: 55). 
En esta nueva razón gubernamental, el interés se va a concebir en términos más abiertos y complejos. Es, en realidad, un entramado de expectativas individuales y colectivas, sociales y económicas, mercantiles y públicas, que deben ser manejadas, puestas en equilibrio. En la gubernamentalidad liberal, el gobierno "es algo que manipula intereses" (Ibid.). Para poder ser gubernamentalizable, el sujeto, individual o colectivo, se va a constituir, correlativamente, en sujeto de interés.

Ciertamente, el principio de utilidad es tan obvio, tan fundamental al discurso de la evaluación que esta resulta inconcebible sin aquel. Casi resulta innecesario argumentarlo. Ian Shaw, en su clásico estudio sobre la evaluación cualitativa, lo señala de modo explícito: "lo que guía la práctica de la evaluación es cierta visión de finalidad. En el centro de esta visión está la idea de que la evaluación tiene una utilidad para las políticas, los servicios y la práctica" (Shaw 2003: 112). Y antes, aunque en un sentido más general, Ernst House ya había identificado la zona de convergencia ideal de los distintos enfoques evaluativos disponibles a finales de la década de 1970 en los principios de competencia y libre elección, que permitirían seleccionar "lo mejor":

"[T]odos los enfoques de la evaluación dan por supuesto un mercado libre de ideas en el que, en un plano ideal, los usuarios seleccionarán lo mejor. Mediante la competición entre las ideas, se fortalece la verdad y se perfeccionan los servicios sociales. (...) [T] odos los enfoques asumen que un mayor conocimiento hace de alguna manera felices, mejores o satisfechas a las personas (...)" (House, 2000 [1980]: 62).

En efecto, encontramos la premisa de la utilidad y su correlato subjetivo, la concepción del sujeto como sujeto de interés, en la base de los diferentes enfoques que constituyen, más allá de su dispersión teórica, el dominio discursivo de la evaluación. Así, lo que busca medir el enfoque sistemático es la función de satisfacción de una utilidad individual, de un interés subjetivo (incrementar la cualificación, salir de la pobreza...), aunque este se determine unilateralmente por la gerencia de la intervención evaluada. Una función de utilidad establecida por agregación, mediante encuesta estadística, de utilidades individuales previamente operacionalizadas, para ser, finalmente, cuantificadas. En los enfoques alternativos (de estudio de caso, constructivista, democrático...), la premisa de la utilidad no varía. Lo que cambia es la instancia que elige, la que decide sobre la utilidad que debe ser objeto de valoración, así como el procedimiento de estimación (más cualitativo y dialógico): el sujeto se concibe en términos colectivos, acentuando el carácter diverso, plural, de los intereses que están en juego en una evaluación, y la necesidad correlativa de una negociación, un diálogo, una deliberación de la cual derivar los juicios evaluativos. 


\subsection{El examen de los efectos}

De lo anterior se sigue que la evaluación proporciona a la práctica gubernamental una verdad factual: la verdad de su utilidad, apreciada empíricamente mediante la observación de sus efectos, de las consecuencias fácticas de la acción de gobierno. Esa verdad se articula sobre una dicotomía básica: la distinción éxito/fracaso, inherente al arte liberal de gobernar, construido a partir del siglo XVIII sobre la base de la economía política. Esta, de acuerdo con Foucault, no considera las prácticas de gobierno desde el punto de vista de su origen legal, moral o religioso, sino desde la óptica de sus efectos: la cuestión consiste, entonces, en "saber qué efectos tiene [una práctica de gobierno] y si éstos son negativos". De este modo, "[e]l éxito o el fracaso reemplazarán (...) la división legitimidad/ilegitimidad". La distinción éxito/fracaso y el examen de los efectos como formas de establecer la verdad de la acción gubernamental se constituyen, así, en principio de autolimitación del gobierno. La "autolimitación por el principio de la verdad (...) [fue] la cuña formidable que la economía política introdujo en la presunción indefinida del Estado de policía” (Foucault 2009: 28-31).

El discurso y las prácticas evaluativas se inscriben perfectamente en este esquema. Como hemos señalado, las evaluaciones producen juicios relativos a la eficacia de la acción de gobierno sobre la base de una observación técnica, mediante la cual se verifica el ajuste entre procesos y efectos, entre medios y fines. Los enunciados evaluativos son, básicamente, juicios (de coherencia, de funcionalidad, de eficacia...) acerca del ajuste entre esas series de objetos. Los desajustes verificados, la constatación técnica de incoherencias, disfuncionalidades e ineficacias en la acción de gobierno, deben, en teoría, conducir a reorientar dicha acción gubernamental, a modificarla, a suprimirla incluso. Así, la distinción éxito/fracaso y el examen de los efectos como formas de establecer la verdad de la acción de gobierno, su autolimitación por el principio de esa verdad, todos estos rasgos, descritos por Michel Foucault como supuestos y condiciones de posibilidad de la gubernamentalidad liberal, constituyen, junto a los principios de utilidad e interés, las condiciones generales de posibilidad de la evaluación como saber instrumental y tecnología de gobierno.

Hay, por tanto, un nexo entre la gubernamentalidad liberal, el desarrollo del discurso evaluativo y la institucionalización de esta práctica. Este nexo se concreta en un vínculo que creemos fundamental: la estrecha relación entre la hegemonía gerencialista persistente en el campo evaluativo y los principios de la llamada Nueva Gestión Pública, inspiradores de las reformas neoliberales de la Administración en Estados Unidos en los años '70 y, algo después, en Europa. En el marco teórico y práctico de la Nueva Gestión Pública, la evaluación va a encontrar un sentido bien definido: ajustar, de acuerdo con criterios de management, la relación coste-resultado en la esfera de la acción de gobierno. Los modelos gerenciales de evaluación han contribuido, así, a apuntalar, teóricamente al menos, la superioridad técnica de los criterios de racionalidad empresarial, 
frente a los principios jurídico-burocráticos, como vectores de organización de la Administración pública neoliberal ${ }^{6}$.

\section{UNA PARADOJA Y DOS EXPLICACIONES}

En el plano teórico, entre el saber evaluativo y las tecnologías liberales de gobierno hay, por tanto, un acoplamiento casi perfecto. Entre evaluación y gubernamentalidad gerencial, entre el discurso evaluativo y esa modalidad específica de neoliberalismo formada por los supuestos y las prácticas de la Nueva Gestión Pública, ese acoplamiento funciona casi como una máquina.

Ciertamente, la evaluación es una forma de poder-saber con funciones y efectos esperados bien definidos: justificación de decisiones sobre la continuidad de las intervenciones públicas en términos de eficacia y eficiencia, reordenación de políticas, racionalización presupuestaria... Sin embargo, el cumplimiento de esos fines se revela, en la práctica, más que dudoso. Como hemos visto, la historia de la evaluación es, en cierto sentido, la historia de su fracaso, el relato de la lucha persistente por dar un uso al conocimiento técnico, por desbloquearlo políticamente: la lucha por "poner la 'teoría en acción"”, en la expresión de Guba y Lincoln (1989: 249). Nos encontramos, así, con un saber técnico convocado para influir en la toma de decisiones públicas que, en la práctica, se muestra irrelevante, carente de uso efectivo.

Esta constatación, una suerte de paradoja, contradice la idea de un arte de gobernar fundado sobre la distinción éxito/fracaso y, en definitiva, sobre la verdad de la técnica. ¿Cómo explicar esta contradicción? Podemos apuntar dos explicaciones (a modo de hipótesis generales), basadas en dos marcos teóricos diferenciados:

1. La primera se enmarca en la concepción foucaultiana de las relaciones entre discursos y prácticas de gobierno: el inevitable desacoplamiento entre éstos y la correlativa superposición de lógicas gubernamentales que se da en cada periodo histórico.

2. La segunda se basa en la difícil relación entre verdad y política, tematizada por Hanna Arendt: más allá de todo desacoplamiento contingente entre discursos y prácticas, la relación entre verdad y política estaría determinada por una suerte de incompatibilidad fundamental.

${ }^{6}$ Un análisis detallado de las reformas neoliberales del Estado, los postulados de la Nueva Gestión Pública y los rasgos del llamado gobierno empresarial se encuentra en Du Gay (1994, 2000). Sobre la relación entre evaluación y Nueva Gestión Pública, Cfr. González Tejerina (2010) y Rivero (2011). 


\subsection{Foucault: Desacoplamiento y superposición de lógicas gubernamentales}

Entre el discurso y la práctica evaluativas, por un lado, y entre los discursos y las prácticas gubernamentales, por otro, no encontramos una homología estricta, un acoplamiento mecánico. En realidad, siguiendo el tenor de la obra foucaultiana, no lo podemos encontrar. Más bien, como diría Deleuze en su postrera interpretación de Foucault, lo que encontramos es su "anisomorfía", un juego de "insinuaciones recíprocas", de "presiones y capturas mutuas" entre formas que son heterogéneas, irreductibles entre sí (Deleuze 1987: 95-96). Por eso, el saber evaluativo no puede tomarse como una reproducción simple del dispositivo de gobierno que ha solicitado ese saber, que lo ha contratado y espera utilizarlo. Dicho, de nuevo, en términos deleuzianos: "lo enunciable" (los discursos) no es homologable a "lo visible" (los dispositivos, las prácticas), aunque dichas formas puedan insinuarse recíprocamente "como en una batalla" (Ibid.: 95).

Aplicado al objeto que nos ocupa, esto significa que en el interior de los dispositivos de gobierno modernos operan racionalidades, discursos y prácticas más antiguos que la evaluación, el gerencialismo o la Nueva Gestión Pública. Racionalidades que tienen un espacio propio y que lo defienden frente a otras lógicas, frente a otros discursos y prácticas. Para poder materializarse efectivamente, la razón evaluativa debe abrirse camino frente a esas racionalidades previas, que dominan desde hace tiempo dos campos de prácticas diferenciables:

En el campo estratégico de las decisiones gubernamentales, la aplicación del saber evaluativo está en conflicto con una razón política concernida por juegos de intereses que se sitúan más allá de lo técnico. Aunque los postulados de la evaluación o de la razón gerencial hayan sido asumidos formalmente, tanto en Estados Unidos como en Europa, por los decisores públicos, el problema de estos no es siempre, ni mucho menos, la eficacia o la eficiencia de las políticas. Estos criterios, constantemente invocados, son desplazados en la práctica por otras consideraciones (electorales, mediáticas, estratégicas...). La racionalidad evaluativa es una racionalidad técnica que está en relación de tensión con una racionalidad político-partidista que tiende a ser hegemónica en la toma de decisiones públicas.

En el campo operativo de las prácticas de gestión y control de la Administración, hay una razón jurídico-burocrática heredada que sigue siendo dominante. La práctica evaluativa y, con ella, la razón gerencial o el discurso de la Nueva Gestión Pública, tratan de abrirse camino desplazando a la "vieja" burocracia de cuño jurídico. Pero, en un entorno en el que esta razón jurídico-burocrática es dominante, en el que imperan el ajuste a Derecho Administrativo y la reproducción del régimen legal-procedimental que le es propio, la evaluación no dispone de un espacio viable, ni de un tiempo oportuno. Las prácticas de gestión de las intervenciones públicas están supeditadas a instancias de control jurídico-financiero que se imponen sobre los funcionarios de modo apremiante. Son instancias 
dotadas, por su propia naturaleza, de una eficacia legal de la que la evaluación carece ${ }^{7}$.

Estos ejes de tensión, estos frentes abiertos por otras racionalidades y otras prácticas, obstruyen la materialización efectiva de la evaluación, más allá de las proclamas gerencialistas y de la invocación oficial de los principios de la eficacia y la eficiencia. La evaluación es, por tanto, sólo un discurso y una práctica más, y quizá la menos vinculante, la menos perentoria. Un discurso que circula en un espacio ocupado ya por otras racionalidades. En este entorno policéntrico, los usos de la evaluación van a ser mínimos.

En las prácticas de gobierno, encontramos, de este modo, toda una superposición de racionalidades que se enfrentan o, en ocasiones, se respaldan mutuamente. En este juego de ajustes y desacoplamientos se inscriben el discurso y la práctica evaluativas. Un juego que evoca las palabras con las que Michel Foucault daba por concluido, el 4 de abril de 1979, su curso dedicado a la genealogía del liberalismo:

"[E]n el mundo moderno, (...) toda una serie de racionalidades se encabalgan, se apoyan, se rebaten, se combaten unas a otras. Arte de gobernar en la verdad, arte de gobernar en la racionalidad del Estado soberano, arte de gobernar en la racionalidad de los actores económicos y, de manera más general, arte de gobernar en la racionalidad de los mismos gobernados (...). ¿Qué es la política, en definitiva, si no el juego de esas diferentes artes de gobernar con sus diferentes ajustes y, a la vez, el debate que ellas suscitan?" (Foucault 2009: 310).

\subsection{Arendt: verdad y política}

$\mathrm{Si}$, en el interior de ese "Arte de gobernar en la verdad" que limita la acción de gobierno en virtud del principio de eficacia, el conocimiento (la verdad de la técnica), no se utiliza, ello podría deberse a algo que está más allá del desacoplamiento entre discursos y prácticas gubernamentales o de la superposición de racionalidades que "se encabalgan, se apoyan, se rebaten, se combaten unas a otras". Es la relación misma entre verdad y política lo que, más allá de las contingencias históricas, se caracteriza por el desajuste y el extrañamiento mutuo. Una relación determinada por una suerte de discordancia fundamental: la verdad y la política son, siempre lo han sido, difícilmente compatibles.

${ }^{7}$ Esta es una de las conclusiones de nuestra investigación sobre la evaluación en España, en la que, cuando los funcionarios entrevistados hablan sobre dicha actividad, su discurso expresa inmediatamente un conflicto entre la teoría y la experiencia: la evaluación no despliega efectos; se mantiene como una especie de "ideal de la buena gestión pública". Es, además, un deber exógeno, derivado de las exigencias de la Unión Europea. Unas exigencias menos vinculantes que las derivadas de las instancias de control jurídico-administrativo, que son las que realmente se imponen con el rigor propio de la ley (Rivero 2015: 541-542). 
Para Hannah Arendt, la exclusión mutua entre verdad y política es obvia: "Nadie ha dudado jamás que la verdad y la política nunca se llevaron demasiado bien, y nadie (...) puso nunca la veracidad entre las virtudes políticas" (Arendt 2016: 347). De este modo, el "compromiso con la verdad (...) se siente como una actitud antipolítica" (Ibid.: 366). La razón fundamental de tan radicales apreciaciones reside en la naturaleza coactiva de los juicios de verdad: " $L a$ verdad implica un elemento de coacción" (Ibid.), que interfiere con la política de dos formas:

1. En primer lugar, es evidente que la verdad, en tanto que instancia coactiva, puede entrar en conflicto con los intereses de grupos políticos diversos y, por supuesto, obstaculizar decisiones gubernamentales ${ }^{8}$.

2. En relación con lo anterior, aunque en un sentido más fundamental, la naturaleza coactiva de la verdad distorsiona el espacio de discusión y acuerdo que, para Arendt, constituye el ámbito esencial de la política: los juicios de verdad están "más allá del acuerdo, la discusión, la opinión o el consenso" (Ibid.: 367).

El conflicto entre verdad y política se corresponde, entonces, con una antítesis clásica: la que opone la verdad a la opinión, equiparable esta última con la persuasión y la retórica. Dado que "la opinión y no la verdad está entre los prerrequisitos indispensables de todo poder" (Ibid.: 357), la antítesis verdad/ opinión deriva en conflicto verdad/política. La verdad es, en este sentido, antipolítica o, cuanto menos, extrapolítica: "tiene su fuente en un lugar que no es el campo político y (...) es tan independiente de los deseos y anhelos de la gente como lo es la voluntad del peor de los tiranos" (Ibid.: 368).

En el plano de las estrategias discursivas que conforman la práctica de la discusión política, esta antítesis se manifiesta en la degradación de los hechos al estatus de opiniones: cuando los hechos, "las verdades factuales incómodas", se oponen al interés del gobierno, o de los distintos grupos que entran en confrontación en el campo político, "se las transforma en opiniones" (Ibid.: 362). Se reproduce así, sin denotaciones metafísicas, la impotencia platónica del hombre veraz que, al regresar del mundo de las ideas y tratar de comunicar su verdad a la multitud, la ve "desaparecer en la diversidad de puntos de vista (...), y caer hasta el espacio incierto de la opinión" (Ibid.: 363). De este modo, la disolución de la demarcación entre hecho y opinión constituye una estrategia discursiva para "negar o tergiversar cualquier clase de verdad" (Ibid.), una forma de protegerse de la fuerza coactiva de la verdad cuando esta se opone al propio interés.

\footnotetext{
${ }^{8}$ Arendt asume la distinción (leibniziana) entre verdades de razón (formadas por axiomas matemáticos y descubrimientos científicos) y verdades de hecho (factuales), constituidas por actos y acontecimientos que son "el producto invariable de los grupos de hombres que viven y actúan juntos" (Arendt 2016: 353). Dado que estos actos y acontecimientos "constituyen la textura misma del campo político" (Ibid.), son principalmente las verdades de hecho las que entran en conflicto con el poder, con pocas posibilidades de sobrevivir a su "embestida feroz" (Ibid.: 355).
} 
Arendt es categórica respecto a la relación entre opinión y política: la fortaleza de todo gobierno descansa en la opinión de los gobernados, en el número de los que comparten la misma opinión, pues "ni siquiera el gobernante más autocrático o tirano podría llegar jamás al poder, y menos aún conservarlo, sin el apoyo de los que tuvieran una mentalidad semejante" (Arendt 2016: 357). Quizá sean generalizaciones un tanto excesivas. En cualquier caso, en el mundo moderno, en ese tiempo en el que Michel Foucault establece el nacimiento de la gubernamentalidad liberal, la idea de un gobierno concernido por la opinión de los gobernados resulta decisiva. Si aceptamos la interpretación foucaultiana, el arte liberal de gobernar se hace inteligible, no solo en virtud del principio de autolimitación por la verdad, sino, asimismo, por su atención a los intereses de los gobernados. Este "interés en plural", que se manifiesta superficialmente en la opinión, debe ser manejado, puesto en equilibrio por el gobierno: en la gubernamentalidad liberal, recordémoslo, el gobierno es "algo que manipula intereses" (Foucault 2009: 56). El énfasis en la relación entre gobierno y opinión introduce, entonces, un elemento de tensión en el núcleo de la gubernamentalidad liberal: tensión entre gobernar de acuerdo a la naturaleza de las cosas, a la distinción éxito/fracaso, a la autolimitación por el principio de la verdad, por un lado; gobernar descansando sobre la opinión, persuadiendo a los muchos, manipulando intereses, por otro. Salvo que concedamos a las opiniones el estatus de verdades, parece evidente que la limitación de la acción de gobierno por el principio de la verdad poco tiene que ver con la persuasión, la manipulación de intereses o la fuerza del número de los que comparten una opinión.

Y así, en la época contemporánea, el gobierno que descansa sobre la opinión -y, correlativamente, sobre la persuasión y la retórica- deriva, según Arendt, en uso político de la falsedad deliberada. Esta práctica, que lleva al límite el conflicto entre verdad y política, corre a cargo de especialistas técnicos, asesores gubernamentales que desarrollan dos variedades de lo que sería, lisa y llanamente, un "arte de la mentira" (Arendt 2015:14):

Por un lado, "la mentira aparentemente inocua de los especialistas de relaciones públicas" (Ibid.), cuyo origen está en la formación de la sociedad de consumo de masas. Estos especialistas en publicidad transforman la relación entre gobernantes y gobernados en relación con audiencias, con públicos objetivo a los que vender mensajes: "la relación entre representante y elector se transforma en la relación propia de vendedor y comprador" (Arendt 2013: 459). Los especialistas publicitarios al servicio del gobierno operan, en efecto, "con opiniones (...), con la disposición a comprar, esto es, con intangibles cuya realidad concreta es mínima" (Arendt 2015:15). La premisa con la que trabajan es psicológica: "la manejabilidad humana", que se vende como un producto más "en el mercado de la opinión" (Ibid.). Para estos especialistas, "la mitad de la política es fabricación de imágenes y la otra mitad el arte de hacer creer en las apariencias" (Ibid.). El gobierno moderno, "algo que manipula intereses" en la interpretación foucaultiana, es pura manipulación de las mentes, para Arendt.

La segunda forma que adopta la mentira en la política contemporánea es más sutil. Tiene por sujeto al experto en resolución de problemas, el problem 
solver, llamado a orientar científicamente la toma de decisiones políticas. Puede que sea algo excesivo calificar de "arte de la mentira" las prácticas de estos expertos, como hace Arendt. Sería más prudente señalar su desinterés deliberado por lo real concreto, la disolución de los hechos en la abstracción formal de sus métodos: los problem solvers van a tratar de "hallar fórmulas, preferiblemente expresadas en lenguaje pseudomatemático, que unificaran los fenómenos más dispares con los que les enfrentaba la realidad" (Arendt 2015: 17). Las técnicas de estos asesores gubernamentales constituyen métodos de sustitución/eliminación de lo real: "diferentes maneras de traducir calidades y contenidos en cantidades y números con los cuales calcular resultados, que después (...) jamás llegaron a producirse" (Ibid.: 34) ${ }^{9}$. Los problem solvers viven, de este modo, "en un mundo desasido de los hechos" (Ibid.: 33). Para hacer su trabajo no necesitan "hechos ni información"; parten de un modelo previo, "tenían una 'teoría' y todos los datos que no encajaban en esta eran negados o ignorados" (Ibid.: 36). Instalados en tal marco cognitivo, sus técnicas no pueden ser otra cosa que "métodos de desasimiento de los hechos" (Ibid.: 37). Métodos coherentes, a su vez, con una política que desprecia la realidad: "El apartamiento de los hechos y la técnica de la solución de problemas fueron recibidos porque el desprecio a la realidad era inherente a la política" (Ibid.: 38).

En definitiva, todo este dispositivo de asesoramiento formado por especialistas en publicidad y expertos en resolución de problemas es posible porque los objetivos políticos son casi exclusivamente psicológicos; su propósito es "crear un específico estado mental" (Ibid.: 35). Y esta forma de hacer política es consistente, casi como si se tratara de su precondición técnica, con la manipulación sistemática de lo real concreto, operada mediante la aplicación de métodos de análisis pseudomatemáticos cuyo rasgo fundamental es "la incapacidad o la repugnancia a consultar de la experiencia y aprender de la experiencia" (Ibid.: 38).

¿Pero qué relación tienen, si es que tienen alguna, las reflexiones arendtianas sobre los usos del conocimiento y la mentira en política con nuestra descripción precedente del discurso y la práctica de la evaluación? Creemos que existe una conexión fundamental, en primer término histórica. Una conexión que nos permite proyectar lo que acontece en el campo evaluativo más allá de los límites de esta disciplina.

En efecto, la valoración que hace Arendt de la política norteamericana a finales de la década de 1960 encaja perfectamente con lo que sucede en el ámbito de la evaluación en esas mismas fechas, en las que tiene lugar su expansión

${ }^{9}$ Arendt construye su valoración de las prácticas de los problem solvers a partir del estudio de los llamados Documentos del Pentágono, la Historia del Proceso de Formulación de Decisiones de los Estados Unidos acerca de la Política del Vietnam, encargada por el Secretario de Defensa, Robert McNamara, en 1967 y filtrados al New York Times en 1971. El estudio de los Documentos del Pentágono sirvió a Arendt para elaborar una reflexión general sobre la falsedad deliberada en la política contemporánea. Como veremos, esta reflexión no resulta extraña a lo que acontece en el campo de la evaluación en los Estados Unidos en esas mismas fechas. 
político-administrativa. El paralelismo cronológico no es casual, sino que encierra otras dos convergencias:

1. La institucionalización de la evaluación en Estados Unidos se produce, recordemos, a partir de 1965. Su fin expreso fue determinar los efectos de los programas de guerra contra la pobreza, desarrollados por la Administración Johnson. Estos programas discurrieron en paralelo a la guerra del Vietnam. Las decisiones sobre ambas guerras, la interior (social, metafórica) y la exterior (militar, real) estuvieron ligadas y muy concernida por cuestiones de opinión e imagen ${ }^{10}$.

2. El asesoramiento técnico desplegado para orientar la toma de decisiones en la guerra del Vietnam como en la guerra contra la pobreza tiene un fundamento metodológico común: el análisis de sistemas.

Este último punto es especialmente significativo: los debates surgidos en el campo de la evaluación en los Estados Unidos desde finales de la década de 1960 se proyectaron sobre el mismo paradigma metodológico (el modelo del análisis de sistemas), generando, con una argumentación epistemológica y metodológica más elaborada, críticas similares a las formuladas, casi intuitivamente, por Arendt respecto a las técnicas aplicadas por los problem solvers en su asesoramiento a los procesos decisionales del conflicto vietnamita ${ }^{11}$ : en las propuestas de Scriven, de Stake y de Eisner, primero, y de Guba y Lincoln, así como de House y Howe, después, encontramos, con mayor desarrollo argumentativo, las mismas críticas al objetivismo cuantitativo, una denuncia similar del desprecio de los hechos derivado de la reducción estadística y su formalismo. Correlativamente, lo que estos autores proponen es, recordemos, una restauración de lo real concreto, una recuperación de la experiencia vivida por los actores. De ahí la apelación al descenso al campo y la realización de evaluaciones naturalistas, el recurso al juicio cualitativo del experto en la cosa evaluada, la restitución del discurso de los sujetos afectados por la evaluación. Y en la misma línea, en clave política, en estos teóricos de la evaluación encontramos una crítica de la falta de uso de las evaluaciones, o de su uso interesado y unilateral con fines de legitimación. Crítica, finalmente, del imperio político y cultural de la imagen en sociedades en las que "el bienestar social está amenazado por el caos y la

${ }^{10}$ Las decisiones tomadas durante la guerra del Vietnam se hicieron de espaldas a la evidencia proporcionada por los observadores sobre el terreno y estuvieron guiadas por imperativos de imagen (crear la opinión de que se estaba ganando la guerra, primero, y que no se estaba perdiendo, después) (Arendt 2015: 21-22). Sobre la relación entre la guerra de Vietnam y la guerra contra la pobreza de Johnson, Cfr. Helsing (2000).

${ }^{11}$ Arendt señala expresamente que los métodos de los problem solvers estuvieron extraídos de del análisis de sistemas (Arendt 2015: 16). Como vimos, el modelo de evaluación elegido por la Administración Johnson para evaluar los programas de guerra contra la pobreza a partir de 1966 fue, en efecto, el análisis de sistemas, en su concreción como enfoque sistemático, que fue transferido desde el Departamento de Defensa al de Salud, Educación y Bienestar en 1965, con el fin de formular y evaluar los programas de guerra contra la pobreza en términos de coste/beneficio (House 2000: 185-186). 
confusión que generan estas nuevas dinámicas de los medios de comunicación de masas" (House y Howe 2001: 179).

Arendt nos ayuda a comprender por qué -o quizá, más simplemente, cómola evaluación, cuyo sentido último es la utilidad, deviene en actividad inútil, finalmente ineficaz. La historia de la evaluación, el relato de esa lucha teórica y metodológica por poner el conocimiento en acción, reproduce la tensión fundamental, muy antigua, entre verdad y política. La razón por la cual la evaluación constituye una práctica sin uso efectivo estaría, si aceptamos las tesis arendtianas, y llevando al límite la superposición de racionalidades planteada por Foucault, en la naturaleza coactiva de los juicios de verdad, en el conflicto que estos plantean con los intereses específicamente políticos, en su interferencia en el espacio de discusión y acuerdo que, para Arendt, sería esencialmente constitutivo de la actividad política.

En la época contemporánea, en el tiempo en que vivimos, esta tensión entre verdad y política se reconfigura en virtud de dos factores que se alimentan mutuamente:

1. Una intensificada dependencia entre gobierno y opinión.

2. Una praxis política que obedece, en alto grado, a imperativos de comunicación e imagen (una política desasida de los hechos, en la expresión arendtiana).

La alineación de estos factores configura un plano inclinado hacia la manipulación sistemática de la información y, en el límite, hacia la falsedad deliberada. Las prácticas de especialistas en publicidad y problem solvers, denunciadas por Arendt, y las prácticas de los evaluadores, cuestionadas por ellos mismos (en tanto que críticos de la evaluación), se deslizan por ese mismo plano. Un plano que conduce a eso que hoy llamamos posverdad.

\section{DISCUSIÓN FINAL}

Arendt decía que la degradación de los hechos al estatus de opiniones era una práctica común en el debate político contemporáneo. Y es probable que así sea. Pero también se da el proceso inverso y recíproco: el uso interesado de la verdad por parte de gobiernos, partidos o grupos de presión; el intento de presentar como verdades de hecho lo que, en virtud de su fuerza coactiva, pueda servir de justificación técnica a decisiones ya pensadas y asumidas (ese aura de legitimidad que, según Weiss, otorgaba la evaluación a los programas evaluados). El poder, en sus diversas manifestaciones, exhibe entonces su verdad, su propia forma de legitimación basada en los hechos, el despliegue de una "ideología de la evidencia" (Augè 2004: 176). No creemos que sea muy osado decir que el principio tecnocrático del one best way sirve a menudo para sacar del espacio de la discusión política cuestiones que se presentan bajo la forma, técnicamente coactiva, de una única respuesta. 
Diluir la verdad en la opinión o enfatizar su fuerza coactiva: lo que habría, en definitiva, es un juego estratégico en el que la verdad se usa políticamente como argumento imperativo y, alternativamente, la opinión se usa como argumento contra la coacción de la verdad, cuando esta se opone al propio interés. En estas estrategias en las que está en juego el estatus de las cosas, presentándolas como hechos o como opiniones, se inscribirían los problemas relativos a la producción/recepción de los informes técnicos (evaluativos o de otra clase), al déficit de uso del conocimiento que estos proporcionan o su empleo con fines de legitimación. Lo que acontece en una estructura de acción política caracterizada, como apuntaba Michel Foucault, por la superposición de racionalidades e instancias de decisión que se rebaten o se apoyan entre sí. En ese entrecruzamiento de racionalidades, sobredeterminado por la contraposición radical entre verdad y política, no parece que sea la razón técnica, el gobierno limitado por el principio de la verdad, lo que se impone en la práctica sino, como Arendt señalaba, su distorsión: la eliminación de la experiencia al servicio de la creación de estados mentales específicos. En esta hipótesis, la abstracción formal y la sofisticación estadística albergarían, aun sin pretenderlo, la posibilidad de un uso perverso: serían, en efecto, métodos de desasimiento de los hechos; la precondición técnica de una política desasida de los hechos.

Una última cuestión, para concluir. Hablar de una política desasida de los hechos es, hoy, hablar de política post-factual, de política de la posverdad, en definitiva. Es casi inevitable concluir este artículo con una reflexión en torno a esta cuestión: ¿cómo se inscribe la relación entre gubernamentalidad, evaluación y verdad, y, en sentido amplio, el problema del uso político del conocimiento, en esta especie de paradigma perverso que se cubre hoy bajo el término posverdad?

Lo nuevo en la cuestión de la posverdad no es que antes se gobernara en la verdad y ahora no. Lo nuevo, desde el punto de vista del emisor, el "político posverdad", es su carencia de escrúpulos, su falta de pudor al realizar afirmaciones indiferentes o directamente contrarias a cualquier evidencia. El desasimiento de los hechos alcanza así su grado máximo, en una especie de momento cínico en el que el desprecio ante las evidencias (técnicas, científicas o de cualquier tipo) se exhibe ya sin disimulo. Pero este desprecio inmoderado por los hechos no acontece sin que también haya algo nuevo en las audiencias. Desde el punto de vista del receptor, lo que importa es que ese discurso post-factual refuerce sus valores y creencias, que confirme sus aspiraciones. Lo cual, a su vez, acontece en un contexto de crisis del sistema político, de polarización intensificada de los posicionamientos ideológicos y partidistas, de grupalidad cerrada en silos y articulada en comunidades virtuales ${ }^{12}$.

En este contexto altamente polarizado, en el que todo suceso lleva asociado un significado político, ideológico o moral, la correspondencia de un enunciado con los hechos pierde valor argumentativo. Si lo que importa para otorgar fuerza perlocutiva al discurso es su correspondencia con los fines y valores por los que emisor y receptor previamente han optado, la verdad factual deja de servir como

${ }^{12}$ Para una revisión multidisciplinar de la cuestión, Cfr. Jordi Ibáñez (2017). 
fundamento argumentativo y decisional; lo que queda es su simulacro. La verdad que importa, la que legitima y provoca adhesiones, de haber alguna verdad invocada, ha de ser de otro tipo: acaso sea una verdad moral, cuya supuesta superioridad justificaría cualquier medio, cualquier mentira ${ }^{13}$. La evaluación de políticas, los informes técnicos o la evidencia científica devienen, en cualquier caso, una suerte de "hipótesis inútil". Algunas prácticas apuntan, significativamente, a la supresión misma de la evaluación como disciplina auxiliar en el proceso de formulación de políticas públicas ${ }^{14}$.

La cuestión, eminentemente prescriptiva, de las posibles salidas a esta situación, excede el propósito de este artículo. Solo nos atrevemos a sugerir la conveniencia de revisar las propuestas, sin duda utópicas, de House y Howe acerca de la evaluación democrática deliberativa. Porque, incluso aceptando, con Gianni Vattimo, que la ausencia de verdad sea la precondición de la democracia ${ }^{15}$, no podemos aceptar que cualquier afirmación sea válida. Despertar del sueño de la verdad-objeto, de la verdad entendida como absoluta correspondencia entre el enunciado y la cosa, no significa renunciar a la discusión racional y al examen de la veracidad de las afirmaciones, en un marco de deliberación compartido.

\section{REFERENCIAS BIBLIOGRÁFICAS}

ARENDT, H. (2013): Sobre la revolución, Madrid, Alianza.

-(2015): "La mentira en política. Reflexiones sobre los Documentos del Pentágono", en Crisis de la república, Madrid, Trotta, pp. 347-402.

-(2016): "Verdad y política" en Entre el pasado y el futuro. Ocho ejercicios sobre la reflexion política, Barcelona, Península, pp. 347-402.

AUGÈ, M. (2004): ¿Por qué vivimos? Por una antropología de los fines, Barcelona, Gedisa.

CRONBACH, L. (1963): “Course Improvement through Evaluation”, Teachers College Record, 64, pp. 672-683.

DELEUZE, G. (1987): Foucault, Barcelona, Paidós.

DU GAY, P. (1994): “Making up managers: bureaucracy, enterprise and the liberal art of separations", British Journal of Sociology 45 (4), pp. 655-674.

-(2000): In Praise of Bureaucracy. Weber, Organization, Ethics, Londres, Sage.

EISNER, E. (1979): The Educational Imagination, New York, Macmillan.

13 Sobre los usos de la verdad y la moralización de la política propia de los movimientos neoconservadores, Cfr. Franzé (2011).

${ }^{14}$ En su ensayo sobre la falsedad en la era Trump, Michiko Kakutani describe cómo el presidente de los Estados Unidos "se saltó sistemáticamente el engranaje habitual de elaboración de programas políticos y el proceso normal de análisis y revisión (...): se decidía algo en función de lo que la Casa Blanca o el Congreso de mayoría republicana querían que sucediera, y luego trataban de dar su explicación o mostrar las ventajas de la decisión" (Kakutani 2019: 29).

15 "[E]l adiós a la verdad es el inicio, y la base misma, de la democracia. Si existiera una verdad 'objetiva' de las leyes sociales y económicas (...), la democracia sería una elección por completo irracional: sería mejor confiar el Estado a los expertos, a los reyes-filósofos de Platón o a los premios Nobel de todas las disciplinas" (Vattimo, 2010: 18). 
FOUCAULT, M. (2009): Nacimiento de la biopolítica. Curso del Collège de France (1978-1979), Madrid, Akal.

FURUBO, J. E., SAHNDAL, R. y RIST, R. (eds.) (2000): The evaluation atlas. Seventeen countries profiles, New Brunswick, Transaction.

GONZÁLEZ TIJERINA, S. A. (2010). "Evaluación, herramienta clave en la Administración pública”, SEE-valuación, Año 3, 10, pp. 29-33.

GUBA, E. G. y LINCOLN, B. S. (1986): "The Countenances of Fourth Generation Evaluation: Description, Judgement, and Negotiation", Evaluation Studies Review Annual, Vol. 11, pp. 70-80.

-(1989): Fourth Generation Evaluation, Newbury Park, Sage.

HELSING, J. W. (2000): Johnson's War/Johnson's Great Society: The Guns and Butter Trap, Westport, Praeger.

HOUSE, E. R. (1979): “The objectivity, fairness, and justice of federal evaluation policy as reflected in the Follow Through evaluation", Educational Evaluation and Policy Analysis, Vol. 1, pp. 28-42.

-(2000): Evaluación, ética y poder, Madrid, Morata.

HOUSE, E. R. y HOWE, K. R. (2001): Valores en evaluación e investigación social, Madrid, Morata.

IBÁÑEZ FANÉS, J. (ed.) (2017): En la era de la posverdad. 14 ensayos, Barcelona, Calambur.

KAKUTANI, M. (2019): La muerte de la verdad. Notas sobre la falsedad en la era Trump, Barcelona, Galaxia Gutemberg.

MACLAUGHLIN, M. W. (1975): Evaluation and Reform. Cambridge, MA, Ballinger.

MERINO, M. (2010): Fundamentos de evaluación de políticas públicas, Madrid, Agencia Estatal de Evaluación de las Políticas Públicas y Calidad de los Servicios.

RIVERO, A. (2011): “Tensiones y problemas de legitimación en la evaluación de políticas públicas. Un análisis a la luz del caso español", Revista Enfoques, Ciencia Política y Administración Pública, Vol. IX, nº 14, pp. 195-224.

-(2015): Más allá de la eficacia: Crítica y hermenéutica de la evaluación. Tesis doctoral (inédita). Madrid, UNED.

RIVLIN, A. M. (1971): Systematic Thinking for Social Action, Washington, DC, Brookings Institution.

ROSSI, P. H., FREEMAN, H. E. y WRIGHT, S. R. (1979): Evaluation. A Systematic Approach, Beverly Hills, Sage.

SCRIVEN, M. (1972): "Objectivity and subjectivity in educational research", en Philosophical Redirection of Educational Research, Chicago, National Society for the Study of Education, pp. 94-142.

-(1973): “Goal free evaluation", en School Evaluation: The Politics and Process. Berkeley, McCutchan, pp. 319-328.

-(1976): "Evaluation biass and its control", en Evaluation Studies Review Annual, Vol. 1, pp. 119-139.

SHAW, I.F. (2003): La evaluación cualitativa. Introducción a los métodos cualitativos, Barcelona, Paidós.

SIMÓN, M. A. (2011): "La moralización de la política en el neoconservadurismo", en Política y verdad, Madrid, Plaza y Valdés, pp. 209-224.

SMITH, E. y TYLER, R. W. (1942): Appraising and Recording Student Progress, New York, Harper \& Row.

STAKE, R. E. (1967): “The countenance of educational evaluation”, Teachers College Record, 68, pp. 523-540. 
-(1976). Evaluating Educational Programmes: The Need and the Response, Washington, OECD Publications Center.

-(1978): “The case study method in social inquiry”, Educational Researcher, 7, pp. 5-8.

TYLER, R. W. (1950): Basic Principles of Curriculum and Instruction, Chicago, University of Chicago Press.

URTEAGA, E. (2010). "La evaluación de políticas públicas en el mundo", SEE-valuación. Año 3, 10, pp. 34-44.

VATTIMO, G. (2010): Adiós a la verdad, Barcelona, Gedisa.

WEISS, C. (1980): "Knowledge creep and decision accretion", Knowledge, Creation, Diffusion, Utilisation, Vol. 1, n 3, pp. 381-404.

-(1987): "Where politics and evaluation meet", en D. Palumbo (comp.): The Politics of Program Evaluation. Newbury Park, Sage, pp. 47-70. 
